\title{
Archéopages
}

Archéopages Archéologie et société

37 | 04/2013

Jardins

\section{Le monde en son jardin. Débat}

Propos recueillis par Catherine Chauveau

Marie-Hélène Bénetière, Frédérique Boura, Amina-Aïcha Malek et Catherine Chauveau

\section{(2) OpenEdition}

Journals

Édition électronique

URL : https://journals.openedition.org/archeopages/352

DOI : 10.4000/archeopages.352

ISSN : 2269-9872

Éditeur

INRAP - Institut national de recherches archéologiques préventives

Édition imprimée

Date de publication : 1 février 2014

Pagination : 62-69

ISSN : $1622-8545$

Référence électronique

Marie-Hélène Bénetière, Frédérique Boura, Amina-Aïcha Malek et Catherine Chauveau, «Le monde en son jardin. Débat », Archéopages [En ligne], 37 | 04/2013, mis en ligne le 01 avril 2015, consulté le 21 janvier 2022. URL : http://journals.openedition.org/archeopages/352 ; DOI : https://doi.org/10.4000/ archeopages.352 


\section{Le monde en son jardin}

Il existe une infinité de gradations entre les diverses actions de l'homme

sur le milieu naturel. Cultivés, entretenus, exploités, structurés, ces espaces

anthropisés le sont plus ou moins. Parmi eux, le jardin : un lieu totalement artificialisé créé pour représenter une nature habilement sublimée.

\section{Marie-Hélène Bénetière Frédérique Boura}

\author{
est historienne des jardins, chargée \\ de mission à la direction générale des \\ Patrimoines du ministère de la Culture \\ et de la Communication et ancienne \\ directrice de la rédaction de Polia, \\ Revue de l'art des jardins. Parmi ses \\ dernières publications : avec F. Boura, \\ Jardins en Alsace: quatre siècles \\ d’histoire, Lyon, éditions Lieux-Dits, \\ 2010 ; Jardin. Vocabulaire typologique \\ et technique, coll. Principes d'analyse \\ scientifique, Paris, Editions \\ du Patrimoine, 2000
}

est archéologue, directrice adjointe de la direction régionale des Affaires culturelles de Lorraine. Parmi ses dernières publications : avec M.-H. Bénetière, Jardins en Alsace : quatre siècles d'histoire, Lyon, éditions Lieux-Dits, 2010 ; Les Nouvelles de l'Archéologie 83-84 «Archéologie des jardins », Paris, Errance, 2001.

\section{Amina-Aïcha Malek}

architecte de formation, est chargée de recherche au CNRS à l'UMR 8546 «Archéologie et philologie d'Orient et d'Occident », dans l'équipe Monde romain. Parmi ses dernières publications : Sourcebookfor Garden Archaeology, Methods, Techniques, Interpretations and Field examples, Berne, Suisse, Peter Lang, coll. Parcs et Jardins, 2013 ; “De l'espace pictural à l'espace du jardin : mosaïques et jardins dans les domus de l'Afrique romaine", Archéologie des jardins : analyse des espaces et méthodes d'approches, P. Van Ossel, A.-M. Guimier-Sorbets, M.-C. Marinval (eds), Nanterre, 2010 ; « Prairies et Jardins : Art et Nature dans la domus de l'Algérie antique », in L'Algérie en héritage, art et histoire, catalogue de l'exposition (7 octobre 2003- 25 janvier 2004), Paris, Institut du Monde arabe, Actes Sud, p. 148-156, 2003. 

iti: tintin.

\section{हE्]

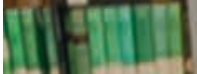 \\ .}

लाM

$-7+$

Fin

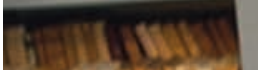

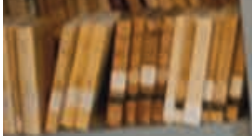

1

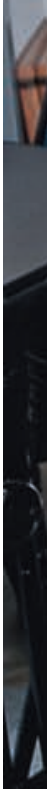

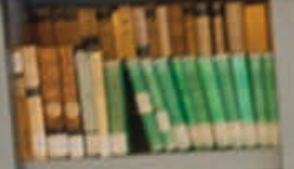

He

for

-

Tivere

III)

Tr.

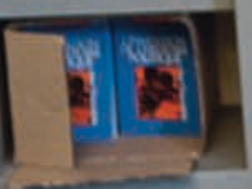

A 1

Fent rin

$-1$

7.
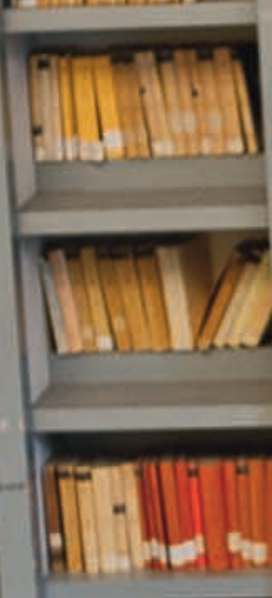

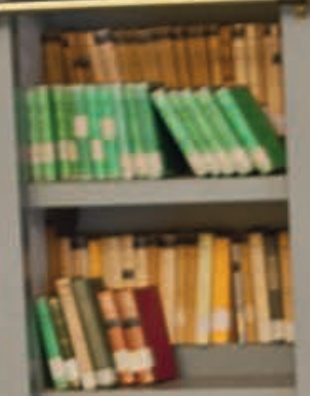

atpolutint

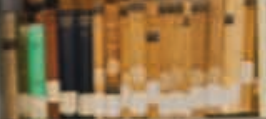

औसमी

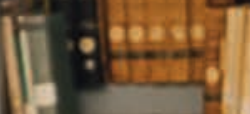
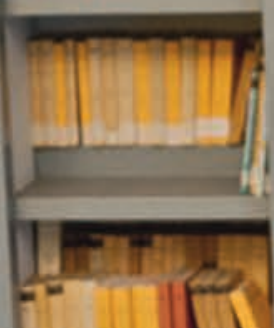

$=-1$
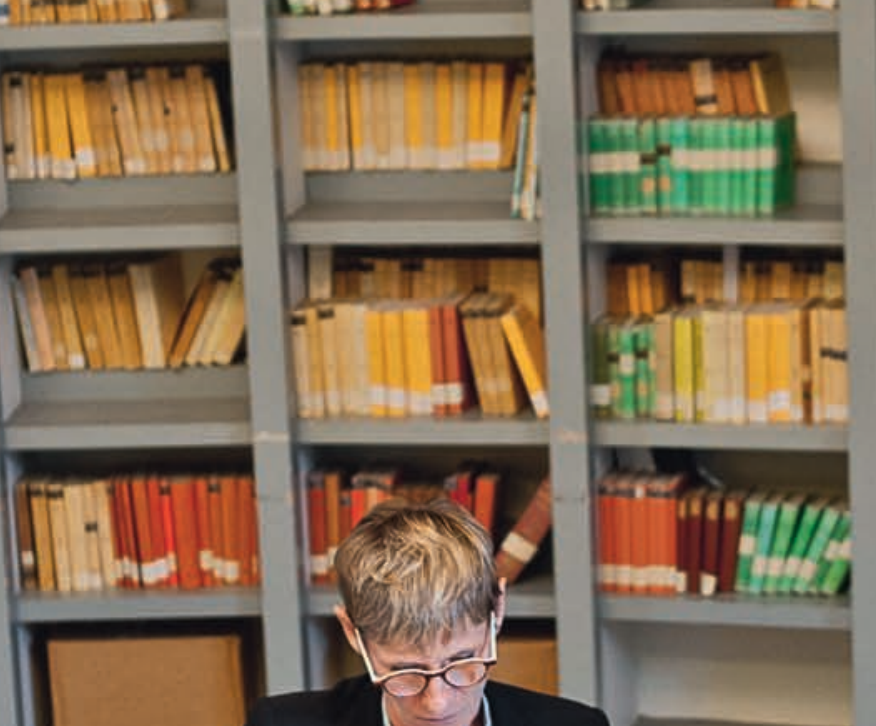
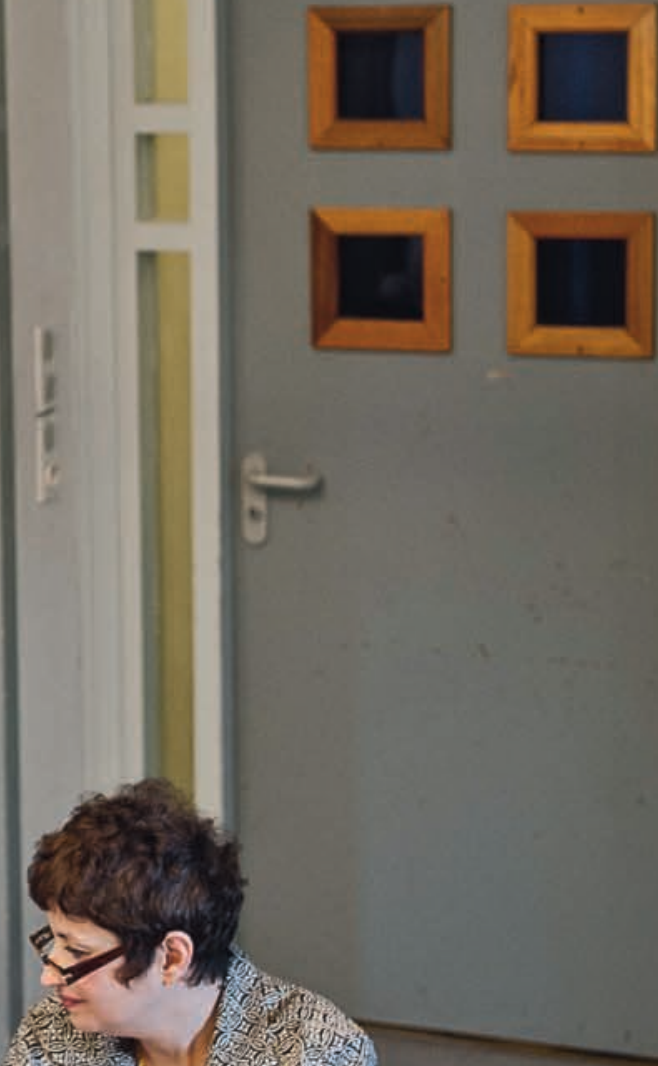

67?

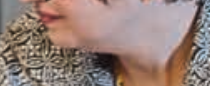


Frédérique Boura En 2001, lorsque nous avions travaillé au numéro pour les Nouvelles de l'archéologie, il était évident que, pour tous les archéologues, un jardin est un objet d'archéologie. Cependant la recherche en ce domaine ne s'est pas autant développée que l'on avait pu le souhaiter. Car la structuration académique des champs d'étude fait que si un projet de recherche sur le jardin ne se rattache pas à des disciplines déjà installées ou à des actions patrimoniales codifiées, il peine à progresser. C'est un cercle vicieux. Si des restaurations ne sont pas prévues, les fouilles des espaces jardins ne sont pas prescrites, et s'il n'y a pas de fouilles, les études et donc les connaissances restent très limitées ; la discipline s'essouffle, les spécialistes se raréfient et les prescriptions s'étiolent. Or tandis que les jardins se transforment en friches ou disparaissent, l'art des jardins se perd peu à peu. Il a par exemple manifestement été « oublié » des années 1920 aux années 1970-80, période où l'on a cru que pour restaurer les grands domaines anciens, il suffisait de planter. Les architectes qui restauraient n'avaient juste pas pris en compte que le parc avait été construit grâce à des systèmes subtils de drainage, qui faute d'entretien, ne fonctionnaient plus, et pour des usages de fréquentation totalement différents de la visite en masse de week-end. Entre l'absence de maîtrise du drainage, le tassement provoqué par les piétons et les voitures etc., le sol et les végétaux étaient profondément perturbés. Le paysage artificialisé ancien était détruit, même pas compris, et le paysage artificialisé actuel ne reposait sur aucun équilibre. Les visiteurs pataugeaient dans les allées, les arbres tombaient, l'herbe se refusait à pousser... Les architectes se sont rendu compte que ces parcs étaient des ouvrages reposant sur un équilibre absolument précaire entre un aménagement de type de génie civil et une construction hyper raffinée.

Marie-Hélène Bénetière La définition que nous avions retenue pour le Vocabulaire des Jardins est « un espace organisé, généralement clos, indépendant ou associé à un édifice, comportant des végétaux d'utilité ou d'agrément, cultivés en plein-terre ou hors sol, créé à partir d'une modification plus ou moins profonde du site naturel...» ${ }^{\mathbf{1}}$. Elle renvoie à des types de jardins très différents et explicite ce qui caractérise le jardin, c'est-à-dire l'intervention humaine sur la nature, profonde et dans la durée : sur le relief, sur le sous-sol, sur le réseau hydrique, sur la couverture végétale... On crée réellement un écosystème ; on construit un espace en trois dimensions en quelque sorte : en sous-sol, en surface et... en se projetant dans l'avenir! Car le jardin n'est pas le même de jour en jour, d'année en année. Dès que l'on arrête de maintenir cet écosystème, le jardin disparaît : il s'ensauvage et rapidement on ne voit même plus sa trace.

Amina-Aïcha Malek L'art des jardins vise à atteindre une nature idéalisée, façonnée selon une culture particulière. Chaque culture propose sa vision d'une nature perfectionnée ou idéalisée qui la sépare des autres terres cultivées ou entretenues. Notre définition se veut universelle, mais les jardins sont historiquement et culturellement spécifiques. Elle attire l'attention sur les dimensions écologiques et biologiques du jardin qui offre ainsi des champs d'étude pour une compréhension de la transformation anthropique de la nature, des espèces vivantes, des adaptations à de nouvelles formes de la culture. Cette recherche dépasse largement l'étude du jardin en soi. L'archéologie des jardins a longtemps été reléguée à l'étude des jardins historiques et à leur restitution. Or la recherche non seulement de leurs configurations en surface mais aussi de leur mise en oeuvre en tant qu'environnements construits ouvre la voie à une nouvelle compréhension des structures profondes du site et de leur intégration dans l'environnement.

F B Il est primordial d'aborder le jardin en conscience de ce statut particulier : c'est un endroit qui fonctionne de façon indépendante par rapport à son environnement naturel tout en restant profondément relié à lui. Son étude se rattache à celle de l'archéologie du paysage, à celle de l'anthropisation des territoires, phénomène de longue durée plus ou moins maîtrisé dont les actions peuvent se révéler plusieurs centaines d'années après, avec des processus de résiliences.... Ces travaux sont invisibles, mais repérables par l'investigation archéologique et ces actions sur l'environnement nous ont parfois surpris par leur ampleur. Au château de Vallery et à La Bâtie d'Urfé, par exemple, Anne Allimant ${ }^{2}$ a pu mettre en évidence qu'avant d'installer le jardin, on avait enlevé des milliers de mètres cubes de terre pour conduire l'eau sur une surface d'argile plus ou moins imperméable et réalisé des systèmes très complexes de drainage « naturel ». À Saint-Cloud, on s'est rendu compte que l'organisation des pièces d'eau et des différents espaces était conçue grâce à une compréhension fine du substrat géologique. C'est aussi à Saint-Cloud que les plans des espaces du jardin ont été organisés de façon à créer une anamorphose. Les paysagers de l'époque moderne déviaient des cours d'eau, recréaient des strates sédimentaires, modifiaient les pentes de terrain, édifiaient des maçonneries sous-terraines et des batteries de moulins, vannes, digues... pour gérer de façon très subtile la circulation de l'eau. Cette maîtrise passe aussi par la gestion des plantations : un aulne, par exemple, peut pomper jusqu'à 900 litres d'eau par jour et par arbre ; donc une aulnaie assainit un terrain et permet, dans les terrains proches, de développer des parterres plus secs, adaptés à d'autres espèces qui n'auraient jamais poussé là spontanément. L'art du jardin apparaît avoir été à la pointe de l'utilisation des savoir-faire traditionnels comme des innovations scientifiques et techniques.

A-AM Ces traditions horticoles, ces connaissances pointues, diverses d'une culture à l'autre, se conjuguent 


\section{Construit mais vivant, le jardin nous confronte} à notre imaginaire et à nos sens.

Amina-Aïcha Malek

1. Extrait de la définition 
avec d'autres composantes culturelles que sont les goûts, les modes... La fouille du patio du palais d'El Hamra, en Espagne, a permis de préciser les différentes phases d'aménagement du jardin de l'époque maure au $\mathrm{Xv}^{\mathrm{e}}$ siècle, très mal documenté par les textes. Il a pu être reconstitué avec son niveau surbaissé par rapport aux allées agrémentées de pelouses interrompues par des plates-bandes fleuries et scandées d'arbres, et avec son grand canal bordé probablement de myrte, comme le mentionne les textes andalous. Ce jardin encaissé disparaîtra au $\mathrm{XV}^{\mathrm{E}}$ siècle pour faire place à un autre, convoquant des pratiques complètement différentes, avec la plantation d'espèces provenant d'Amérique et d'Afrique du sud.

M-H B Oui, les aménagements sont souvent en lien avec l'accroissement du règne végétal connu, grâce aux voyages, et donc aux connaissances botaniques. Car fréquemment ces travaux ont aussi pour but de modifier la nature du substrat pour l'adapter aux espèces choisies. On creuse alors des fosses de plusieurs mètres cubes, sur de très grandes surfaces, qu'on remplit de substrat convenant, par exemple, pour planter des rhododendrons ou des azalées. Certaines essences étaient en telle vogue à certaines périodes qu'on investissait des masses d'argent et réalisait des prouesses pour les avoir ! Il y a eu la manie des peupliers d'Italie au XVIII ${ }^{\mathrm{e}}$ siècle pour donner un caractère méridional à une composition paysagère, et dans la même idée, celle des cèdres. Puis est né l'engouement pour les plantes rapportées par les grandes expéditions maritimes aux XVIII ${ }^{\mathrm{e}}$ et $\mathrm{XIX}^{\mathrm{e}}$ siècles : chênes et conifères américains, par exemple. Les principaux ports de France avaient des jardins conservatoires qui servaient à adapter au climat et la terre locale les plantes qui venaient d'ailleurs. C'était très compliqué d'ailleurs de faire survivre ces végétaux pendant les traversées : il fallait les protéger des rats, du vent, du froid, de l'air salé... Le jardin célèbre la beauté de la nature, certes, mais d'une nature artificialisée, idéalisée. $\mathrm{Au} \mathrm{XVIII}{ }^{\mathrm{e}}$, on est allé jusqu'à planter des arbres morts dans les parcs paysagers pour les faire ressembler à la nature!

F B Mais ce n'est pas pour autant la nature, c'est la projection d'un rêve, en quelque sorte. Les petits « jardins d'allumé », scintillants de coquillages, de bouteilles colorées, de carrelages cassés etc. comme les immenses et fastueux parcs privés puis publics de l'époque moderne sont nés de la même intention : projeter son univers, intime ou social. On pense à ce morceau d'anthologie qu'est la réalisation du jardin dans Bouvard et Pécuchet, où Flaubert dresse la liste des jardins possibles (mélancolique, terrible, majestueux, fantastique, rêveur...) et de tous les éléments nécessaires qu'ils doivent comporter. On y retrouve ces deux dimensions : la conviction intime du beau et le désir de prestige.

M-H B On est dans la concrétisation d'un univers intérieur ou exploré. Le jardin d'Albert Kahn à Boulogne, conçu comme le tour du monde en quatre-vingts pas ; la villa Ephrussi de Rothschild à Saint-Jean-Cap-Ferrat, syncrétisme de la culture italienne, dont le jardin est lui aussi une juxtaposition de jardins à thématiques culturelles... Ce sont des représentations idéales d'un monde idéal dans lequel on vit, on est, on jouit, on expérimente le temps qu'il fait et le temps qui passe.

A-A M Comme l'empereur Hadrien qui a voulu, dans son jardin, représenter le monde tel qu'il le concevait. Les jardins introduits dans les maisons romaines offraient la possibilité de confondre leurs espaces réels avec des espaces imaginaires. Je me suis particulièrement intéressée aux mosaïques de jonchées qui mêlent branches coupées et fleurs de diverses espèces dans un agencement développé jusqu'au bas des murs, sans bordure donc, ce qui est très inhabituel. On est à la fois dans la volonté de représenter quelque chose de réel, la coutume de couvrir le sol de végétaux frais, odorants et colorés, et dans celle de se référer aux productions de l'esprit : l'image du philosophe installé sous un arbre ou près d'une source ; les écrits de Platon, de Lucrèce ; les évocations nostalgiques de l'Âge d'Or, lorsque dieux et humains vivaient ensemble dans les prairies... On est projeté dans quelque chose de plus vaste qui unit les sens et l'esprit. Ces représentations révèlent comment le jardin a la capacité d'engager le visiteur dans un dialogue spatial permanent et renouvelé où espaces imaginaires et espaces réels se confondent et s'articulent. F B Oui, c'est la pratique du jardin qui est essentielle ; le jardin ne se réduit pas à un plan. L'important c'est l'expérience sensorielle du fait de cheminer, de passer de l'ombre à la lumière, d'un espace ouvert à un espace fermé, d'appréhender la demeure. Car le jardin sert aussi à mettre en valeur une demeure. La circulation, souvent un itinéraire « obligé », est tracée pour permettre de déboucher sur un point de vue où la maison sera magnifiée, mise en valeur dans un cadre, un peu comme dans un décor de théâtre avec un premier plan, un second plan, un troisième plan. Dans les grands parcs baroques, la promenade passe par une salle de fraîcheur, un nymphée, un pavillon chinois où l'on va pouvoir boire le thé, par des bosquets... Tout est organisé pour que l'on passe d'une ambiance à une autre. On peut saisir une part de cela par l'archéologie : les tracés, les fosses, les pots, les éléments de réseau hydraulique, le mobilier de jardin, la sculpture ornementale etc. ; tout ceci permet de reconstituer des usages, des pratiques. N'oublions pas que le paysage lui-même participe au décor et à l'expérience de la promenade. Car c'est une expérience physique, comme le montre Jane Austen dans ses romans en décrivant la façon de mettre en valeur une maison. Entendre, regarder, respirer, avoir la sensation du frais, de la lumière, de l'air, de la nonchalance, de l'effort, de la surprise... Tout cela constitue une sorte de préparation 
L'archéologie a montré ce qui est invisible dans le jardin, mais qui le rend possible: la préparation du sous-sol.

Frédérique Boura 
à découvrir, à s'émerveiller. Nous devons le garder à l'esprit pour pouvoir interpréter les structures du jardin et ne pas se contenter de les décrire.

M-H B Oui, pour ne pas passer à côté du sens de ce jardin, il faut s'interroger sur le type d'expérience qu'il propose. Un jour où j'allais visiter un jardin de Soufflot, je suis passée devant une propriété dont le parc était une friche d'où émergeaient deux colonnes torses. C'est grâce aux archives conservées par le propriétaire que j'ai pu comprendre cet endroit dont la mémoire s'était perdue : un parc expiatoire, bâti à grands frais par un contrerévolutionnaire au début du XIX ${ }^{\mathrm{e}}$ siècle afin de remercier Dieu pour avoir échappé à la guillotine. En s'y promenant selon un parcours très strict, on gagnait des indulgences ! L'endroit est d'une infinie complexité, en lien avec l'histoire personnelle du bâtisseur, et ce jusqu'au jardin potager, qui paraissait juste irrégulier alors qu'il était précisément conçu. C'était extraordinaire! Je pense qu'il doit y avoir pas mal de jardins de ce type-là, mais on n'en comprend plus le sens. A-A M Cela me fait penser à une étude de l'anthropologue Catherine Benoit, qui a montré comment des jardins de case en Guadeloupe ne sont pas de simples lieux de production alimentaire ou d'ornement, mais des espaces de protection du corps, cela grâce à la présence de plantes particulières en des endroits spécifiques. Cette organisation est apparue en cartographiant les jardins plante par plante. Cette recherche fait prendre conscience des pistes à suivre pour cerner plus finement ce qu'est un jardin dans des contextes variés. Le jardin est le résultat d'une dialectique constante entre les hommes et la nature.

M-H B Tout à fait. Et beaucoup de ressources qui pourraient être utiles n'ont pas été dépouillées. Dans mon domaine, même sans faire appel à d'autres disciplines, je suis complètement convaincue qu'il reste un superbe travail à faire sur le paysage agricole et les espaces non bâtis à partir des plans-reliefs du XVIII ${ }^{\mathrm{e}}$ et du XIX ${ }^{\mathrm{e}}$ siècles. Il y a des matrices de plans relief qui sont d'une précision que l'on n'imagine pas : la hauteur des haies, la composition des alignements, la couleur des parterres, la variété cultivée dans chacun des champs... Et on peut les rapprocher des archives comptables, très détaillées, de ce travail.

FB Entre ce qui n'a pas été encore exploité, ce qui n'a pas été bien articulé, et la richesse des échanges à susciter entre toutes les disciplines qui l'étudient, le jardin pourrait devenir l'un des grands domaines de recherche des années à venir. 


\section{Le jardin est un état dynamique qui nous échappe.}

Marie-Hélène Bénetière

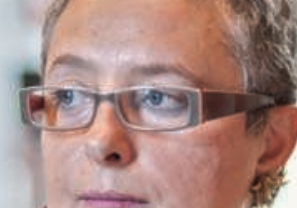

毕

ஜ

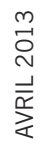
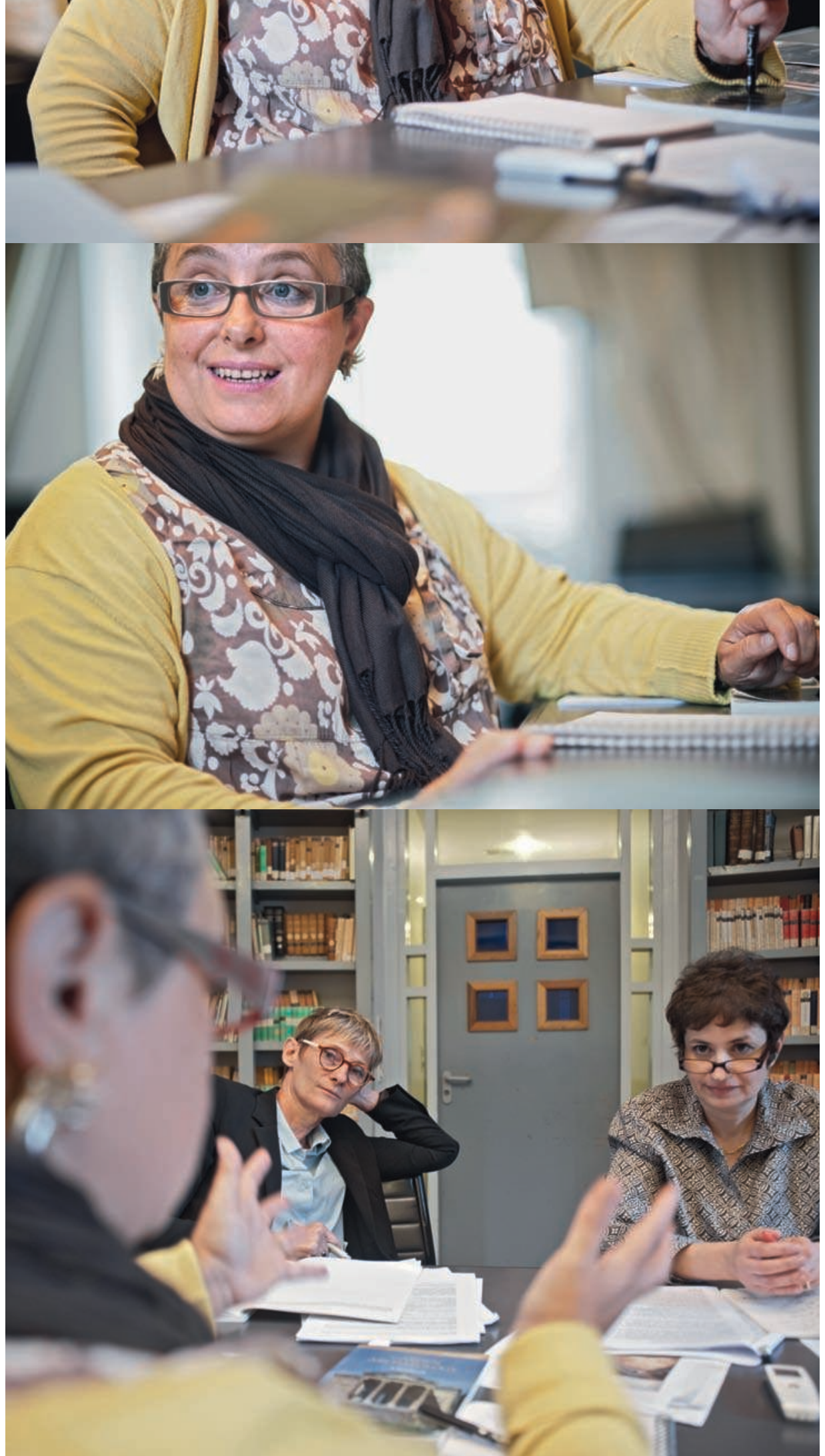